\title{
Macrocytic anemia in Lesch-Nyhan disease and its variants
}

\author{
Hasan F. Cakmakli, MD ${ }^{1}$, Rosa J. Torres, MD PhD ${ }^{2,3}$, Araceli Menendez, MD ${ }^{4}$, \\ Gul Yalcin-Cakmakli, MD ${ }^{5}$, Christopher C. Porter, $\mathrm{MD}^{6,7}$, Juan Garcia Puig, $\mathrm{MD}^{4}$ and \\ H. A. Jinnah, MD PhD ${ }^{6,8,9}$
}

\begin{abstract}
Purpose: Lesch-Nyhan disease is an inherited metabolic disorder characterized by overproduction of uric acid and neurobehavioral abnormalities. The purpose of this study was to describe macrocytic erythrocytes as another common aspect of the phenotype.
\end{abstract}

Methods: The results of 257 complete blood counts from 65 patients over a 23-year period were collected from 2 reference centers where many patients are seen regularly.

Results: Macrocytic erythrocytes occurred in $81-92 \%$ of subjects with Lesch-Nyhan disease or its neurological variants. After excluding cases with iron deficiency because it might pseudonormalize erythrocyte volumes, macrocytosis occurred in $97 \%$ of subjects. Macrocytic erythrocytes were sometimes accompanied by mild anemia, and rarely by severe anemia.

Conclusion: These results establish macrocytic erythrocytes as a very common aspect of the clinical phenotype of Lesch-Nyhan disease and its neurological variants. Macrocytosis is so characteristic that its absence should prompt suspicion of a secondary process, such as iron deficiency. Because macrocytosis is uncommon in unaffected children, it can also be used as a clue for early diagnosis in children with neurodevelopmental delay. Better recognition of this characteristic feature of the disorder will also help to prevent unnecessary diagnostic testing and unnecessary attempts to treat it with folate or B12 supplements.

Genetics in Medicine (2019) 21:353-360; https://doi.org/10.1038/s41436018-0053-1

Keywords: HPRT1; hypoxanthine-guanine phosphoribosyltransferase; Lesch-Nyhan disease; macrocytic anemia; megaloblastic anemia

\section{INTRODUCTION}

Lesch-Nyhan disease (LND, OMIM 308000) is an inherited metabolic disorder caused by deficiency of the enzyme hypoxanthine-guanine phosphoribosyltransferase (HGPRT). This enzyme plays a role in the purine salvage pathway, in which hypoxanthine and guanine are recycled into purine nucleotides. Individuals with LND have a well-described clinical phenotype that includes overproduction of uric acid, behavioral abnormalities, and neurological impairment. ${ }^{1-3}$ In addition to the classic phenotype, there are attenuated variants with overproduction of uric acid, but the neurobehavioral features are milder or even absent. ${ }^{3-5}$ Some reports of LND and its variants have occasionally also mentioned another feature of the phenotype: macrocytic anemia. ${ }^{6,7}$ However, this problem is not widely recognized because it has received very little attention in the literature. There is no information on its frequency, severity, or clinical significance.

In the current study, we summarize longitudinal data from two referral centers where many patients with LND or its variants are evaluated. We also review the world's literature on macrocytosis and/or anemia in LND and its variants. Together, the results show that macrocytosis-with or without anemia-is a nearly universal feature of LND and its neurological variants. In fact, macrocytosis appears to be so common in this population that its absence should raise concern for some secondary process such as iron deficiency. Because macrocytosis is uncommon in unaffected children, it can also be used as a clue for the early detection of LND in children with neurodevelopmental delay of unknown cause. The findings are valuable for raising awareness of this common finding in this disorder, preventing unnecessary diagnostic testing, and reducing unnecessary vitamin supplementation.

\section{MATERIALS AND METHODS}

\section{Review of patient records}

Subjects

After obtaining approval from the local ethics boards, records of subjects diagnosed with LND or its variants were reviewed from two referral centers-La Paz University Hospital in

\footnotetext{
${ }^{1}$ Department of Pediatric Hematology and Oncology, Faculty of Medicine, Ankara University, Ankara, Turkey; ${ }^{2}$ Foundation for Biomedical Research, La Paz University Hospital-IdiPAZ, Madrid, Spain; ${ }^{3}$ Center for Biomedical Network Research on Rare Diseases-ISCIII, Madrid, Spain; ${ }^{4}$ Department of Internal Medicine, Metabolic-Vascular Unit, La Paz University Hospital-IdiPAZ, Madrid, Spain; ${ }^{5}$ Department of Neurology, Faculty of Medicine, Hacettepe University, Ankara, Turkey; ${ }^{6}$ Department of Pediatrics, Emory University School of Medicine, Atlanta, Georgia, USA; ${ }^{7}$ Aflac Cancer and Blood Disorders Center, Children's Healthcare of Atlanta, Atlanta, Georgia, USA; ${ }^{8}$ Department of Neurology, Emory University School of Medicine, Atlanta, Georgia, USA; ${ }^{9}$ Department of Human Genetics, Emory University School of Medicine, Atlanta, Georgia, USA. Correspondence: H A. Jinnah (hjinnah@emory.edu)

The first two authors contributed equally to this work.
}

Submitted 20 March 2018; accepted: 23 April 2018

Published online: 6 June 2018 
Madrid (Spain) and Emory University in Atlanta (USA). All subjects gave written informed consent or verbal assent. All subjects were diagnosed according to currently accepted criteria. $^{2,4}$ Because HGPRT deficiency is inherited as an Xlinked recessive disorder, all cases were males. According to a previous convention regarding the classification of the spectrum of HGPRT deficiency, subjects were divided into three groups. These included classic LND, HGPRT-related neurological dysfunction (HND), and HGPRT-related hyperuricemia (HRH). For LND, the clinical criteria included overproduction of uric acid, self-injurious behavior, neuromotor impairments, and often some degree of intellectual disability. The diagnosis was confirmed by detection of a pathogenic variant in the HPRT1 gene and/or reduced HGPRT enzyme activity in fibroblasts or blood cells. The clinical criteria for HND were the same as for LND, except HND subjects lacked self-injurious behavior. All HND cases had varying degrees of neuromotor impairment, with scores $\geq 5$ on the Burke-Fahn-Marsden dystonia rating scale. The criteria for HRH were the same as for HND, except clinically relevant neuromotor abnormalities were insignificant, with scores $<5$ on the Burke-Fahn-Marsden dystonia rating scale. All subjects who had at least one complete blood count (CBC) were included in the summary statistics, except two who had transfusions that might have influenced the CBC results. These two cases are presented separately.

\section{Hematology studies}

For each subject, we recorded the phenotypic subgroup and age at the time of each $\mathrm{CBC}$. When multiple $\mathrm{CBCs}$ were collected over time, we evaluated each $\mathrm{CBC}$, but used the most recently collected values for the summary tables. CBC parameters recorded included mean corpuscular volume (MCV), hemoglobin, and red blood cell (RBC) count. Many $\mathrm{CBC}$ parameters vary significantly according to age. Macrocytosis is typically defined by RBC with an abnormally large MCV of $>100 \mathrm{fl}^{8}{ }^{4}$ However, the value is lower in children. ${ }^{9}$ For MCV in children, previously published normative curves were used to define normal age-adjusted values, and any values above the ninety-seventh percentile were considered abnormal. ${ }^{9}$ For ages above 20 years, the upper limit of normal was defined according to tradition as $100 \mathrm{fl}$. A subject was considered to have macrocytosis if there was at least one abnormal CBC.

Macrocytosis can occur without anemia. Anemia is typically defined by either low hemoglobin or low hematocrit, which again can vary by age. ${ }^{10}$ For hemoglobin and RBC counts, we used the normal hematology values commonly used in daily practice for children. ${ }^{11}$ Iron deficiency was defined as low ferritin $\left(<12 \mu \mathrm{g} \mathrm{l}^{-1}\right.$ for children $<5$ years old and $<15 \mu \mathrm{g}^{-1}$ for people $>5$ years old) or low transferrin saturation $(<16 \%$ for all ages). ${ }^{12}$

Where available, we also recorded the results for reticulocyte count, vitamin B12, folic acid, erythrocyte folic acid, haptoglobulin, thyroid function tests, direct and indirect bilirubin, erythropoietin levels, serum iron and iron-binding capacity, and lactate dehydrogenase. We also recorded the results of bone marrow biopsy, if available.

\section{Data analysis}

Descriptive statistics are provided in the form of average values \pm standard deviation. All statistical analyses were performed using SPSS 20 https:/www.ibm.com/products/ spss-statistics. Independent samples $t$-tests were used to compare groups. Correlations between MCV and RBC counts were calculated using the Pearson test. Graphs demonstrating age-specific RBC indices were prepared using SigmaPlot version $10 \mathrm{https}$ ///systatsoftware.com.

\section{Review of published literature \\ Selection of reports}

The PubMed database was used to perform a comprehensive search for all previous English-language reports addressing macrocytosis and/or anemia in LND and its variants. The following search terms were used: Lesch-Nyhan or Kelley-Seegmiller, in various combinations with macrocytosis, megaloblastosis, an(a)emia, macrocytic anemia, MCV, and $\mathrm{CBC}$. Other reports were found through the bibliographies of these papers. We also screened individual case reports and case series for reports of hematology studies because these search terms were often omitted from the key word list or abstract.

\section{Hematology studies}

Reports including information about hemoglobin and MCV were included, even when the results only included subjective interpretations, such as "microcytic" or "macrocytic". A total of 15 articles and 2 postgraduate theses encompassing 23 different subjects with LND or its variants were found with at least some comment on hematology parameters (Supplementary Table S1 online). Publications referring to the same cases were combined to avoid redundant data from the same case.

\section{Review of patient records}

\section{RESULTS}

\section{Subjects and $C B C s$}

We retrieved records for 33 subjects from Spain and 32 subjects from the USA who had at least one CBC (Table 1). The Spanish subjects included 19 with LND, 9 with HND, and 5 with HRH. The American subjects included 20 with LND, 10 with HND, and 2 with HRH. Altogether, there were 204 CBCs from Spain and 53 CBCs from America collected over 23 years. The greater number of $\mathrm{CBCs}$ from Spain reflects a regional practice difference. Hematology studies are routinely collected at every clinic visit in Spain because the Mediterranean diet predisposes to iron-deficiency anemia, especially in developmentally disabled children.

\section{Macrocytosis}

Considering all subjects from Spain and the USA combined, macrocytosis was evident in at least one CBC for $92.1 \%$ (35/ 38 ) of LND patients and $83.3 \%(15 / 18)$ of HND patients. 
Table 1 Hematology results from America and Spain

\begin{tabular}{|c|c|c|c|c|c|c|}
\hline & LND & & HND & & $\mathrm{HRH}$ & \\
\hline Country of origin & USA & Spain & USA & Spain & USA & Spain \\
\hline Number of cases & 20 & 19 & 9 & 9 & 2 & 5 \\
\hline Mean age (range) & $18.8(0.3-40)$ & $14.0(1.1-30.5)$ & $29.9(8-67)$ & $31.0(19.5-54)$ & $36(27-45)$ & $16.0(11.5-20)$ \\
\hline Total number of CBCs & 31 & 156 & 19 & 34 & 2 & 14 \\
\hline CBCs with iron parameters & 0 & 145 & 0 & 32 & 0 & 14 \\
\hline
\end{tabular}

Most of these patients had persistent macrocytosis on multiple $\mathrm{CBCs}$ taken over several years, although an occasional $\mathrm{CBC}$ fell within the normal range. Overall, macrocytosis was seen for $84.6 \%$ (159/188) of the total CBCs for LND and 81.1\% (43/53) for HND (Fig. 1a,b). Macrocytosis was not seen in any of 16 CBCs for any of the $7 \mathrm{HRH}$ patients. Only one LND patient and one HND patient with thalassemia trait had microcytosis.

Although $\mathrm{CBC}$ results for infants were not available, macrocytosis could be detected as early as 6 months of age. There were 16 patients with LND below 4 years of age, and 14 had macrocytosis on at least one CBC. There were two HND patients in this age group, and both had macrocytic CBCs. Thus, macrocytosis was typically a very early finding in LND and HND. In some subjects, macrocytosis appeared to worsen with age, although this observation was difficult to confirm because of the changes in normative values over age combined with the paucity of multiple samples from the same subjects over time.

\section{Anemia}

Considering all subjects from Spain and the USA combined, $42.1 \%(16 / 38)$ of LND cases had hemoglobin values below the reference range at least once during the period evaluated (Fig. 2a,b). Among the total $189 \mathrm{CBCs}$ during this period, only 28 revealed anemia. For the HND group, 38.9\% (7/18) cases had anemia at least once. Among the total of 53 CBCs in this group, hemoglobin was low in 14. Only one of seven HRH cases had anemia.

The total RBC count was below normal at least once for $57.9 \%(22 / 38)$ of LND cases, $83.3 \%$ (15/18) of HND cases, and $14.3 \%(1 / 7)$ of HRH cases (Fig. $2 \mathbf{c}, \mathbf{d})$. The total RBC count was below normal for $41.8 \%(74 / 177)$ of CBCs in the LND group, $79.6 \%$ (39/49) of CBCs in the HND group, and 6.2\% $(1 / 16)$ of $\mathrm{CBCs}$ in the HRH group. There was a strong negative correlation between RBC counts and MCV values (coefficient of correlation, $r=0.795, P<0.05$; Fig. 3). Therefore, hematocrit values were less prominently affected (not shown).

\section{Iron status}

Among subjects with LND and HND, nutritional status is sometimes impaired because of difficulties with swallowing, and approximately $30 \%$ receive enteral nutrition. ${ }^{2}$ We therefore paid close attention to laboratory findings related to nutritional deficiencies that could influence CBC parameters. The most common among these is iron deficiency.

For the Spanish cases, 191 of 204 CBCs were accompanied by simultaneous iron measurements because iron deficiency is common among populations following a Mediterranean diet. For the American cases, iron measurements were typically conducted only after the discovery of anemia; thus, CBCs simultaneous with iron measurements were not available. As a result, the influence of iron status was evaluated only for the Spanish cases. Longitudinal analysis showed that 84.2\% (16/ $19)$ in LND group, and $11.1 \%$ (1/9) in HND group experienced iron deficiency at least once. None of the HRH subjects had evidence for iron deficiency. Taking all of the CBCs with iron parameters into consideration revealed that CBCs were collected during an iron-deficient period for 57 of 145 CBCs in the LND group and 1 of 32 CBCs in the HND group.

Because iron deficiency causes microcytosis and might falsely normalize macrocytosis, we further examined the interaction between macrocytosis and iron deficiency. Of 157 CBCs from the Spanish group, only 23 had normocytic values. After excluding CBCs taken during iron-deficient periods ( $n$ $=60)$ and CBCs taken without simultaneous iron parameters $(n=12), 96.6 \%(85 / 88)$ of the CBCs were macrocytic (Fig. 1c, d). Only three $\mathrm{CBCs}$ collected during iron-sufficient periods were normocytic.

\section{Other hematology parameters}

Table 2 summarizes the results of additional hematology studies. For the Spanish LND subjects, reticulocyte counts and vitamin B12 levels were normal in all cases where they were tested. Folate acid levels were low for 6 of 18 patients at least once. In the HND group, three of nine subjects had mild vitamin B12 deficiency and two had low folic acid levels. In the HRH group, one subject had normal vitamin B12 and folic acid. Folic acid supplements did not correct macrocytosis for any case where they were given.

In the American group, all subjects for whom testing was conducted had normal vitamin B12, folic acid, and reticulocyte counts. Homocysteine and methylmalonic acid levels were normal for all three subjects for whom they were collected. Erythrocyte folic acid levels were normal for all six subjects for whom this was evaluated. 

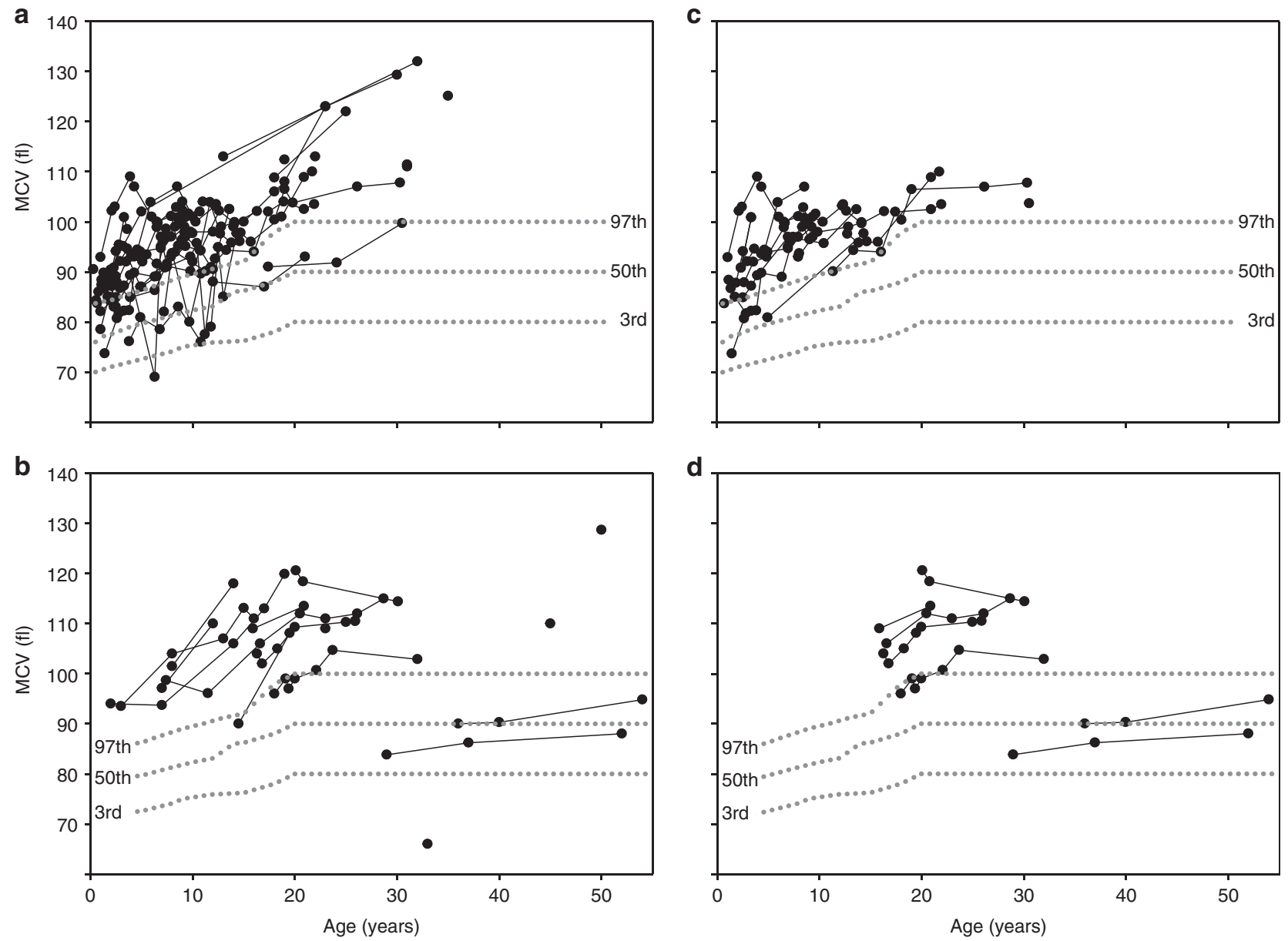

Fig. 1 Erythrocyte volumes as a function of age. (a-d) Mean corpuscular volume (MCV) values for Lesch-Nyhan disease (a and c) and hypoxanthineguanine-phosphoribosyltransferase-related neurological dysfunction (b and $\mathbf{d}$ ). The results from all cases are shown in (a) and (b), whereas the results from only patients with simultaneous normal iron results (to exclude iron deficiency) are shown in (c) and (d). Each circle represents an individual complete blood count result. Circles connected with a line indicate multiple values for the same patient. Age-adjusted normal MCV values are shown as dotted lines at the third, fiftieth, and ninety-seventh percentiles.

\section{Severe anemia}

There were two cases with severe anemia. One Spanish LND case was followed for 18 years and all CBCs showed extreme macrocytosis, reaching $137 \mathrm{fl}$ at 23 years of age. Hemoglobin levels were low from 10 years of age, with the lowest being 7.2 $\mathrm{g} \mathrm{dl}^{-1}$ and resulting in a blood transfusion. This case also had borderline leucopenia $\left(3,500 \mathrm{~mm}^{-3}\right)$. An extensive evaluation revealed normal vitamin $\mathrm{B} 12$, serum and erythrocyte folate levels, haptoglobulin, and iron parameters. The peripheral smear showed anisocytosis, poikilocytosis, tear-drop cells, schistocytes, elliptocytes, dimorphic platelets, and micro and macrocytosis at the same time. With epoetin alfa treatment, anemia improved and hemoglobin levels increased, but not higher than $10 \mathrm{~g} \mathrm{dl}^{-1}$. After withdrawal of this treatment, this case again required blood transfusion. Reintroducing epoetin alfa again increased hemoglobin levels.

A second case with severe anemia from the USA had HND. $\mathrm{He}$ also had macrocytosis from an early age, with hemoglobin values as low as $6.4 \mathrm{~g} \mathrm{dl}^{-1}$, low reticulocyte counts, and mild thrombocytopenia (platelet count: $80,000-100,000 \mathrm{~mm}^{-3}$ ).
Vitamin B12, folic acid, and iron parameters were normal. The erythropoietin level was elevated $\left(2,096 \mathrm{mU} \mathrm{ml}^{-1}\right.$; normal range: 7.3-27.7). He received 6-7 blood transfusions starting from 50 years of age. The bone marrow was normocellular with megaloblastoid hyperplasia in erythroid lineages without any sign of myelodysplastic syndrome. His workup revealed no sign of blood loss via the gastrointestinal system, and ultrasound did not reveal any sign of hypersplenism. However, he had chronic renal failure, which may cause anemia of chronic disease. A younger brother, who also had HND with the same HPRT1 pathogenic variant mutation, had normal renal function with no signs of anemia or thrombocytopenia.

Both of these cases with severe anemia received multiple blood transfusions. Despite extensive workups, a cause for anemia could not be found. There was never any suggestion of immunodeficiency, in terms of frequent or long-lasting infections. These two patients resemble some cases of HGPRT deficiency with severe macrocytic anemia in the literature, although details of the workup of these other cases were not available. $^{13,14}$ 

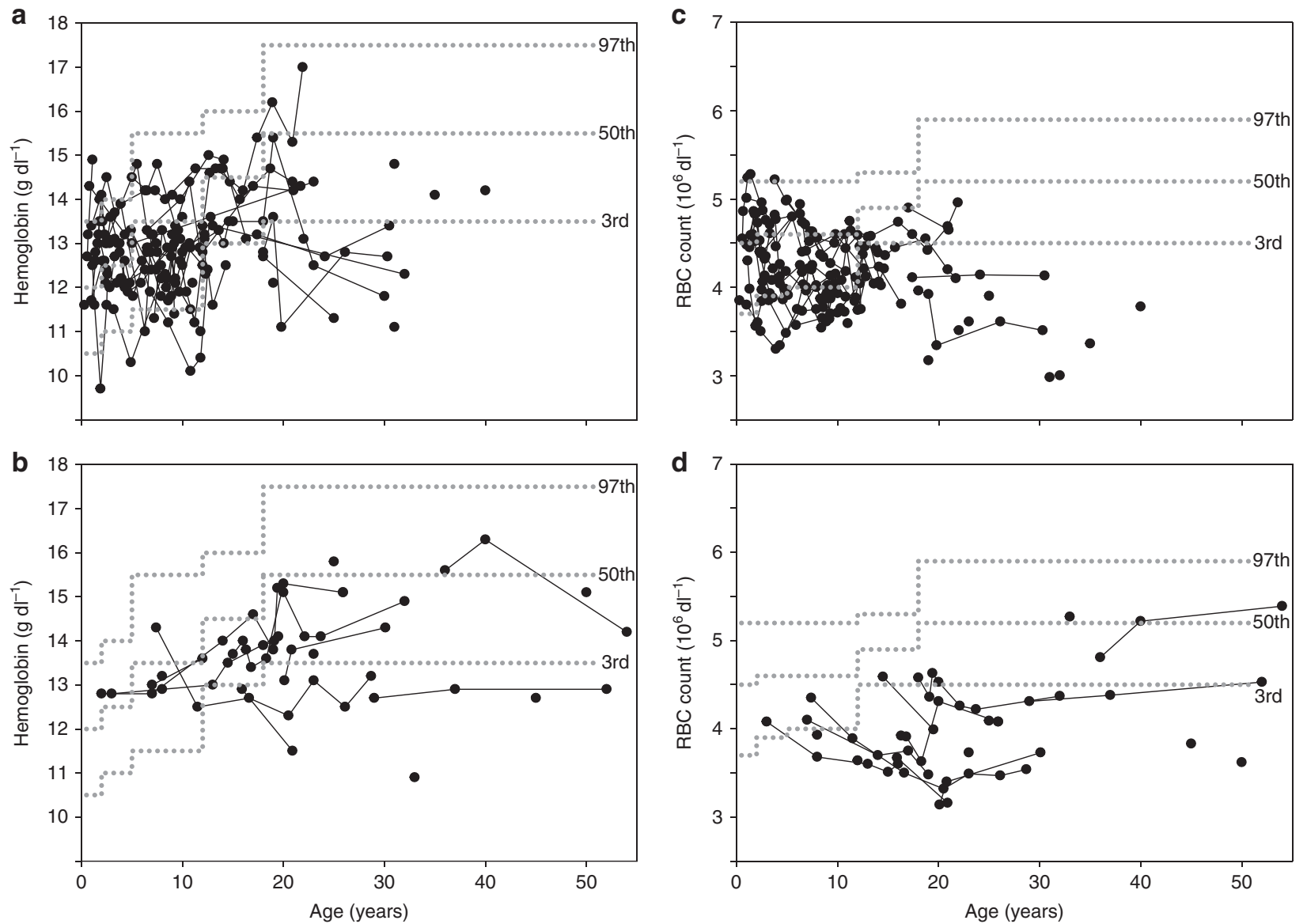

Fig. 2 Total hemoglobin and erythrocyte counts as a function of age. (a-d) Hemoglobin (a and $\mathbf{b})$ and erythrocyte (red blood cell (RBC)) counts (c and $\mathbf{d}$ ) for subjects with Lesch-Nyhan disease ( $\mathbf{a}$ and $\mathbf{c}$ ) and hypoxanthine-guanine-phosphoribosyltransferase-related neurological dysfunction (b and $\mathbf{d}$ ). Each circle represents an individual complete blood count result. Circles connected with a line indicate multiple values for the same patient. Age-adjusted normal values for hemoglobin and RBC counts are shown as dotted lines at the third, fiftieth, and ninety-seventh percentiles.

\section{Review of the published literature \\ Case reports that included $C B C$}

A comprehensive literature review revealed 23 reported cases of LND or its variants with at least 1 CBC (Supplementary Table 1 online). These cases ranged from 3 weeks to 43 years of age. There were 17 cases with LND, and 16 had macrocytosis in at least $1 \mathrm{CBC}(94.1 \%)$. Only six of these cases had anemia, which was usually mild. There were five cases with $\mathrm{HND}$, and all were reported to have macrocytosis without anemia. Vitamin B12 was tested for eight cases, and all were normal. Folic acid was tested for nine cases, and two were considered low. Bone marrow examinations were performed for four LND and three HND cases. All were reported to show erythroid megaloblastosis, although details were not provided.

There was only one patient with $\mathrm{HRH}$, who had normocytic anemia. ${ }^{15}$ However, this patient also had chronic renal failure, which is associated with microcytic or normocytic anemia and may therefore falsely normalize macrocytosis.

Additional descriptions of hematology studies were found in two graduate thesis reports from Nijmegen University. ${ }^{16,17}$ These two reports included 61 cases of HGPRT deficiency,

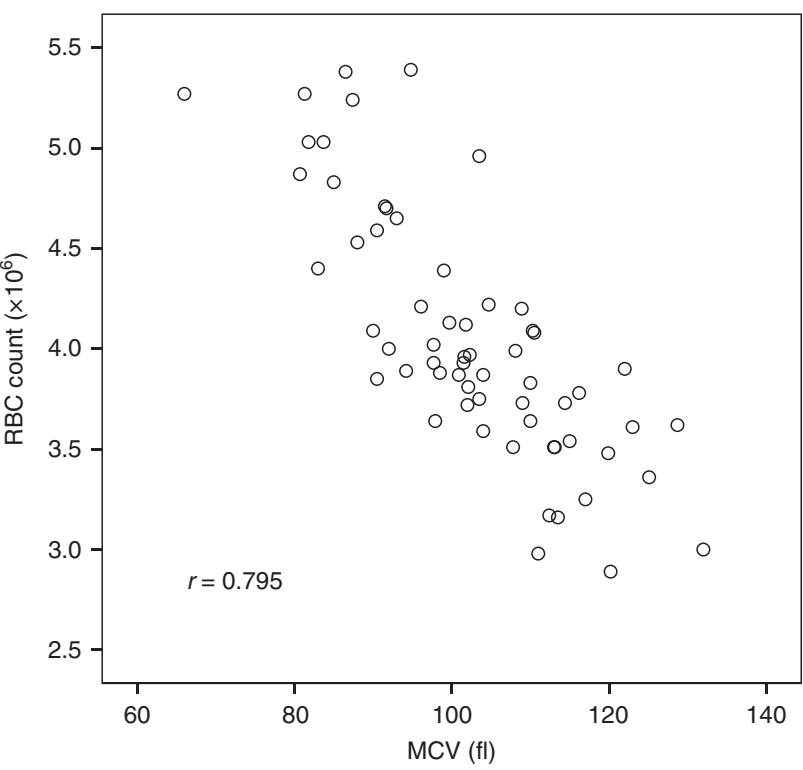

Fig. 3 Correlation of erythrocyte counts versus erythrocyte volumes. Erythrocyte numbers (red blood cell (RBC) count) plotted against the corresponding mean corpuscular volume (MCV) for all cases. 
with $\mathrm{CBC}$ results for 32. Among these cases, 11 had severe anemia, sometimes leading to blood transfusion. Of the cases with severe anemia, seven were reported to be megaloblastic, two were normocytic, one was microcytic, and one was undetermined. One of the seven cases with megaloblastic anemia was reported to respond to folic acid, and another to adenine.

The long-term natural history of LND was described for another series of ten cases in Japan. ${ }^{18}$ This article included a schematic figure showing macrocytic anemia beginning as early as the first year of life, and continuing through adulthood. However, no CBC data were included. Overall, the published literature confirms that macrocytosis-sometimes with anemia-is common in HGPRT deficiency.

\section{DISCUSSION}

These studies show that macrocytic erythrocytes-with or without anemia-are very commonly observed in routine CBCs from subjects with LND and its neurological variants. Nutritional deficiencies were also common in this patient population-particularly iron. When iron deficiency was excluded, macrocytosis was nearly universal. Because data were obtained from two expert centers and a comprehensive review of the literature, the findings are not likely to be restricted to specific populations. Furthermore, data were collected over a wide age range, making the findings relevant for adults and children of all ages. Macrocytosis was absent in subjects with overproduction of uric acid but no neurobehavioral abnormalities (HRH). Overt anemia was less common across the whole spectrum of HGPRT deficiency. When anemia occurred, it was typically mild. Although severe anemia may occur, it appears to be uncommon.

\section{Clinical relevance of macrocytosis}

Macrocytosis is typically defined by an abnormally large MCV of $>100 \mathrm{fl}$. However, this value applies only to adults over 20 years of age. There is a steady increase in the MCV from 6 months to 20 years of age (Fig. 1), but the normal limits are often reported by hematology laboratories in discontinuous age blocks. The lower limit is $84 \mathrm{fl}$ up to 1 year of age, $85 \mathrm{fl}$ up to 3 years of age, $87 \mathrm{fl}$ up to 7 years of age, $90 \mathrm{fl}$ up to 11 years of age, $94 \mathrm{fl}$ up to 16 years of age, and $100 \mathrm{fl}$ after the age 20 years. ${ }^{9}$ Because these discontinuous age blocks do not follow the more continual graded changes with age, macrocytosis is easy to overlook in routine $\mathrm{CBC}$ reports.

Macrocytosis is most commonly caused by a deficiency of vitamin B12 or folic acid, hypothyroidism, or liver disease. It can also be caused by certain drugs, such as alcohol, valproic acid, phenytoin, isoniazide, trimethoprim/sulfamethazole, atovaquone/proguanil, antiretroviral drugs, methotrexate, and some chemotherapy drugs (purine antimetabolites and hydroxyurea). ${ }^{19}$ Less common etiologies include reticulocytosis, leukemia, myelodysplastic or myeloproliferative syndrome, and bone marrow failure syndromes. Extreme macrocytosis $(\mathrm{MCV}>130 \mathrm{fl}$ ) has been reported rarely in association with vitamin B12 deficiency, folate deficiency,
Table 2 Results from additional hematology studies

\begin{tabular}{|c|c|c|c|}
\hline & LND & HND & $\mathrm{HRH}$ \\
\hline $\begin{array}{l}\text { Vitamin B12 (211-911 pg } \\
\mathrm{ml}^{-1} \text { ) }\end{array}$ & $\begin{array}{l}762 \pm 384 \\
(n=24)\end{array}$ & $\begin{array}{l}504 \pm 487 \\
(n=11)\end{array}$ & $535(n=1)$ \\
\hline $\begin{array}{l}\text { Folic acid (3.1-17.5 ng } \\
\left.\mathrm{ml}^{-1}\right)\end{array}$ & $\begin{array}{l}10.8 \pm 6.5 \\
(n=21)\end{array}$ & $\begin{array}{l}10.9 \pm 5.9 \\
(n=12)\end{array}$ & $6.4(n=1)$ \\
\hline LDH $\left(200-400 \mathrm{UI}^{-1}\right)$ & $\begin{array}{l}272.2 \pm 75.9 \\
(n=14)\end{array}$ & $\begin{array}{l}241 \pm 32.9 \\
(n=6)\end{array}$ & $\begin{array}{l}332.7 \pm \\
99.1(n=3)\end{array}$ \\
\hline TSH $\left(0.55-4.68 \mu \mathrm{IU} \mathrm{ml}^{-1}\right)$ & $\begin{array}{l}2.7 \pm 1.2(n \\
=12)\end{array}$ & $\begin{array}{l}2.4 \pm 0.9(n \\
=8)\end{array}$ & $2.4(n=1)$ \\
\hline $\begin{array}{l}\text { Free T4 (0.89-1.72 ng } \\
\left.\mathrm{dl}^{-1}\right)\end{array}$ & $\begin{array}{l}1.2 \pm 0.2(n \\
=12)\end{array}$ & $\begin{array}{l}1.1 \pm 0.1(n \\
=8)\end{array}$ & $1.2(n=1)$ \\
\hline $\begin{array}{l}\text { Haptoglobin }(30-200 \mu \mathrm{g} \\
\left.\mathrm{dl}^{-1}\right)\end{array}$ & $\begin{array}{l}125 \pm 73(n \\
=11)\end{array}$ & $\begin{array}{l}124 \pm 53(n \\
=6)\end{array}$ & NRA \\
\hline Iron $\left(20-150 \mu \mathrm{g} \mathrm{dl}^{-1}\right)$ & $\begin{array}{l}65.3 \pm 29.5 \\
(n=19)\end{array}$ & $\begin{array}{l}108.6 \pm 38.5 \\
(n=9)\end{array}$ & $\begin{array}{l}85.2 \pm 11.8 \\
(n=5)\end{array}$ \\
\hline $\begin{array}{l}\text { Ferritin }\left(<5 \mathrm{yr} ;<12 \mu \mathrm{gl}^{-1}\right) \\
\left(>5 \mathrm{yr}:<15 \mu \mathrm{I}^{-1}\right)\end{array}$ & $\begin{array}{l}64.1 \pm 68.2 \\
(n=19)\end{array}$ & $\begin{array}{l}187.8 \pm \\
122.3(n= \\
9)\end{array}$ & $\begin{array}{l}60.8 \pm 22.8 \\
(n=5)\end{array}$ \\
\hline TFS (>16\%) & $\begin{array}{l}18.7 \pm 8.9 \\
(n=19)\end{array}$ & $\begin{array}{l}36.6 \pm 14.0 \\
(n=9)\end{array}$ & $\begin{array}{l}21.6 \pm 5.0 \\
(n=5)\end{array}$ \\
\hline Reticulocytes (0.5-2.0\%) & $\begin{array}{l}0.96 \pm 0.52 \\
(n=9)\end{array}$ & $\begin{array}{l}1.25 \pm 0.26 \\
(n=3)\end{array}$ & NRA \\
\hline
\end{tabular}

This table includes all available results from all cases from Spain and the USA combined. The numbers of data points varied according to the measurement procedure and patient group, and are shown in parentheses after each result. Normal reference ranges for each measure are shown in parentheses in the first column.

HND hypoxanthine-guanine-phosphoribosyltransferase-related neurological dysfunction; HRH HGPRT-related hyperuricemia; $L D H$ lactate dehydrogenase; LND Lesch-Nyhan disease; NRA no result available; T4, thyroid hormone; TFS transferrin saturation; TSH thyroid stimulating hormone.

antiretroviral therapy for human immunodeficiency virus, hemolytic anemia, and hydroxyurea treatment. ${ }^{20}$ The detection of macrocytosis or anemia in a CBC in a patient with LND often leads to tests to rule out more commonly recognized causes. The results from the current study argue that these tests may not be necessary in most cases. In fact, attempts to treat macrocytosis with vitamin B12 or folate in the absence of overt deficiency do not appear to reverse it. The knowledge that macrocytosis is a common feature of these disorders may be useful to reduce unnecessary testing or the intake of ineffective vitamin supplements in this population.

Macrocytosis is evident from the earliest ages evaluated in LND and its neurological variants. Conditions with macrocytosis in infancy, combined with neurodevelopmental delay, include B12 deficiency, Down syndrome ${ }^{21}$ and the pathogenic variants KLF13 (ref. 22) and ZFYVE20. ${ }^{23}$ The current study suggests that HGPRT deficiency should be added to the list of possible causes when unexpected macrocytosis is found in a routine $\mathrm{CBC}$ from a child with neurodevelopmental disability. In fact, macrocytosis may provide one of the earliest clues for the diagnosis of LND in a patient with unexplained neurodevelopmental delay because $\mathrm{CBCs}$ are routinely performed at all ages. By itself, macrocytosis lacks the specificity and sensitivity to serve as a definitive diagnostic 
tool, but alerts the clinician to the need for more specific testing for HGPRT. In this regard, macrocytosis is similar to uric acid, which is included in many standard clinical chemistry reports, and can provide an early clue for HGPRT deficiency. In fact, the coincidental findings of macrocytic erythrocytes along with elevated uric acid produce a very high level of suspicion, although this suspicion must still be confirmed by tests more specific to HGPRT, such as enzyme assay or HPRT1 gene testing.

\section{Relevance of nutritional deficiencies}

Macrocytosis appears to be so common in LND and HND that its absence should prompt consideration of factors that may falsely normalize it, most notably iron-deficiency anemia and (less likely) thalassemia, chronic diseases, lead intoxication, and others. Conversely, a baseline of macrocytosis may mask the diagnosis of iron deficiency. Thirteen out of 15 subjects with iron deficiency in the current series would have been missed using the standard screening tests of hemoglobin or MCV typically recommended for developing children. For diagnosing iron deficiency among patients with LND or HND, an expanded laboratory panel should be used, including a $\mathrm{CBC}$, a reticulocyte count, serum iron, total ironbinding capacity, and serum ferritin.

Iron deficiency typically causes microcytic anemia and may falsely normalize MCV values for patients with LND and HND. Iron deficiency is very common in chronically debilitated patients, most frequently due to nutritional deficiencies caused by difficulties with solid food consumption. In a study of patients with cerebral palsy, hypochromic anemia was found in $33 \%$, and iron deficiency in $38 \% .{ }^{24}$ In patients on liquid diets, anemia was found in $87 \%$. In our study, $84 \%$ (16/ 19) of LND patients showed iron deficiency, and this high rate is probably related to feeding problems with subsequent nutritional deficiencies. The lower prevalence of iron deficiency in the HND group where feeding is less affected $(11 \% ; 1 / 9)$ supports this hypothesis. In view of these observations, patients with LND and HND should have routine iron measurements (serum iron, serum iron-binding capacity, transferrin saturation, and ferritin), especially if there are known to have feeding difficulties. The CBC alone is insufficient for detecting iron deficiency because baseline macrocytosis obscures the detection of more typical microcytic anemia.

\section{Biological mechanisms for macrocytosis}

Our main goal was to call attention to the clinical factors relating to macrocytosis and anemia in LND; thus, the underlying biological mechanisms were not evaluated. However, it is interesting to speculate on these mechanisms. Only a few published studies have addressed this issue. One evaluated hemoglobin turnover by assessing the incorporation of $\left[{ }^{15} \mathrm{~N}\right]$ delta-aminolavulinic acid or $\left[{ }^{15} \mathrm{~N}\right]$ glycine into bilirubin in a single subject with LND. ${ }^{25}$ The authors concluded that macrocytic anemia was caused by ineffective erythropoiesis and increased turnover of erythrocytes, although the molecular mechanisms were not elucidated.
Another study evaluated the growth of bone marrow cells from two patients with LND and one with HND. ${ }^{26}$ Bone marrow samples from all three cases generated fewer colonies that grew smaller compared with normal individuals. The mechanisms for poor growth were not determined. The authors noted that purines in bone marrow cells are heavily dependent on recycling via HGPRT because of an inherently low capacity for de novo purine synthesis, ${ }^{27}$ and they speculated that HGPRT-deficient marrow cells grew poorly because they were starved for purine nucleotides. ${ }^{7,26}$ Others have suggested that the microtubular cytoskeleton might be disrupted due to guanosine triphosphate deficiency, leading to abnormally shaped erythrocytes. ${ }^{28}$

Several studies have reported purine nucleotide measurements in LND erythrocytes, but a comprehensive review disclosed no consistent changes. ${ }^{29}$ Some studies reported decreases in guanine nucleotides with normal adenine nucleotides, while other studies reported changes in adenine nucleotides with no changes in guanine nucleotides. Thus, purine nucleotide deficiency has not been empirically established as a cause of macrocytosis. Even if we accept the idea that purine nucleotide deficiency may occur, the mechanisms by which such deficiency could cause macrocytosis remain to be determined.

Macrocytosis appears to be unique to erythroid lineages because cells in other tissues, such as brain neurons or skin fibroblasts, are not abnormally large. Macrocytic anemia does not appear to reflect a global failure of the bone marrow because leukocytes or platelet numbers are usually normal, and recurrent infections or excessive bleeding are not common. However, marrow specimens from one LND case showed macromyelocytes, implying potential involvement of the myeloid series as well. ${ }^{26}$

Cell size is normally very precisely regulated, reflecting a tight balance between cell division and increases in cell mass. ${ }^{30}$ In erythroblasts, a size-sensing mechanism may trigger cell division. ${ }^{31}$ It seems feasible that purine nucleotide deficiency slows DNA replication more than it slows the growth of other cellular constituents. If this is the case, the lag in cell division with respect to increases in cell mass may result in abnormally large cells. In this regard, vitamin B12 and folate deficiency are thought to cause macrocytic anemia by reducing purine nucleotides for DNA synthesis. ${ }^{32}$ Furthermore, chemotherapy agents (purine antimetabolites and hydroxyurea) that cause macrocytic anemia also reduce intracellular purine nucleotides. These observations suggest that HGPRT deficiency may lead to purine nucleotide deficiency in bone marrow cells. If this is the case, it could be possible to treat macrocytic anemia through the administration of purine precursors that bypass HGPRT.

\section{ELECTRONIC SUPPLEMENTARY MATERIAL}

The online version of this article (https://doi.org/10.1038/s41436018-0053-1) contains supplementary material, which is available to authorized users. 


\section{ACKNOWLEDGMENTS}

This work was supported in part by the Lesch-Nyhan Syndrome Children's Research Foundation and European Regional Development Fund, and grants from the Fondo de Investigacion Sanitaria del Instituto de Salud Carlos III $(15,10000)$ and Mutua Madrileña Foundation. H.F.C. was supported by Turkish Scientific Council Postdoctoral Research Fellowship grant 2219 and a Turkish Hematology Council HAUD award during his stay in the USA.

\section{DISCLOSURE}

The authors declare no conflicts of interest.

\section{REFERENCES}

1. Fu R, Ceballos-Picot I, Torres RJ, et al. Genotype-phenotype correlations in neurogenetics: Lesch-Nyhan disease as a model disorder. Brain. 2013;137:1282-303.

2. Jinnah HA, Visser JE, Harris JC, et al. Delineation of the motor disorder of Lesch-Nyhan disease. Brain. 2006;129:1201-17.

3. Puig JG, Torres RJ, Mateos FA, et al. The spectrum of hypoxanthineguanine phosphoribosyltransferase deficiency: clinical experience based on 22 patients from 18 Spanish families. Medicine. 2001;80:102-12.

4. Jinnah HA, Ceballos-Picot I, Torres RJ, et al. Attenuated variants of Lesch-Nyhan disease. Brain. 2010;133:671-89.

5. Fu R, Chen CJ, Jinnah HA. Genotypic and phenotypic spectrum in attenuated variants of Lesch-Nyhan disease. Mol Genet Metab. 2014;112:280-5.

6. Van der Zee SP, Schretlen ED, Monnens LA. Megaloblastic anemia in the Lesch-Nyhan syndrome. Lancet. 1968;1:1427.

7. McKeran RO. Factors in the pathogenesis of the brain damage and anaemia in the Lesch-Nyhan syndrome. Ciba Found Symp. 1977;48:83-96.

8. Kaferle J, Strzoda CE. Evaluation of macrocytosis. Am Fam Physician. 2009;79:203-8.

9. Dallman PR, Siimes MA. Percentile curves for hemoglobin and red cell volume in infancy and childhood. J Pediatr. 1979;94:26-31.

10. Looker AC, Dallman PR, Carroll MD, Gunter EW, Johnson CL. Prevalence of iron deficiency in the United States. JAMA. 1997;277:973-6.

11. Lanzkowski P, Lipton J, Fish J Lanzowski's Manual of Pediatric Hematology and Oncology. 6th ed. Cambridge (MA): Academic Press; 2016.

12. Zimmermann MB, Hurrell RF. Nutritional iron deficiency. Lancet. 2007;370:511-20.

13. Hou JW. Atlantoaxial suluxation with recurrent consciousness disturbance in a boy with Lesch-Nyhan syndrome. Acta Paediatr. 2006:95:1500-4.

14. Van der Zee SPM, Lommen EJP, Trijbels JMF, et al. The influence of adenine on the clinical features and purine metabolism in the Lesch-Nyhan syndrome. Acta Pediatr Scand. 1970;59:259-64.
15. Kassimatis TI, Simmonds HA, Goudas PC, et al. HPRT deficiency as the cause of ESRD in a 24-year-old patient: a very rare presentation of the disorder. J Nephrol. 2005;18:447-51.

16. Van der Zee, SPM. Het Lesch-Nyhan syndrom de invloed van adenine op het klinishch beeld en het gestoorde purinemetabolisme [dissertation]. Nijmegen: Nijmegen University; 1972.

17. Lommen, EJP. Hypoxanthine-guanine phosphoribosyltransferase deficiency [dissertation]. Nijmegen: Nijmegen University; 1973.

18. Mizuno T. Long-term follow-up of ten patients with Lesch-Nyhan syndrome. Neuropediatrics. 1986;17:158-61.

19. Veda P. Evaluation of macrocytosis in routine hemograms. Indian J Hematol Blood Transfus. 2013:29:26-30.

20. Takahashi N, Kameoka J, Takahashi N, et al. Causes of macrocytic anemia among 628 patients: mean corpuscular volumes of 114 and 130 $\mathrm{fl}$ as critical markers for categorization. Int J Hematol. 2016;104: 344-57.

21. Roizen NJ, Amarose AP. Hematologic abnormalities in children with Down syndrome. Am J Med Genet. 1993;46:510-2.

22. Spielmann M, Reichelt G, Hertzberg $C$, et al. Homozygous deletion of chromosome $15 q 13.3$ including CHRNA7 causes severe mental retardation, seizures, muscular hypotonia, and the loss of KLF13 and TRPM1 potentially cause macrocytosis and congenital retinal dysfunction in siblings. Eur J Med Genet. 2011;54:e441-5.

23. Stockler $\mathrm{S}$, Corvera $\mathrm{S}$, Lambright $\mathrm{D}$, et al. Single point mutation in rabenosyn-5 in a female with intractable seizures and evidence of defective endocytotic trafficking. Orphanet J Rare Dis. 2014;9:141.

24. Papadopoulos A, Ntaios G, Kaiafa G, et al. Increased incidence of iron deficiency anemia secondary to inadequate iron intake in institutionalized, young patients with cerebral palsy. Int I Hematol. 2008:88:495-7.

25. Samson D, Halliday D, Nicholson DC, et al. Quantitation of ineffective erythropoiesis from the incorporation of $\left[{ }^{15} \mathrm{~N}\right]$ delta-aminolaevulinic acid and $\left[{ }^{15} \mathrm{~N}\right]$ glycin into early labelled bilirubin. II. Anaemic patients. $\mathrm{Br} J$ Haematol. 1976;34:45-53.

26. McKeran RO, Howell A, Andrews TM, et al. Observations on the growth in vitro of myeloid progenitor cells and fibroblasts from hemizygotes and heterozygotes for complete and partial hypoxanthine-guanine phosphoribosyltransferase (HGPRT) deficiency, and their relevance to the pathogenesis of brain damage in the Lesch-Nyhan syndrome. J Neurol Sci. 1974;22:183-95.

27. Lajtha LG, Vane JR. Dependence of bone marrow cells on the liver for purine supply. Nature. 1958;182:191-2.

28. Schneider W, Morgenstern E, Reimers HJ. Disassembly of microtubules in Lesch-Nyhan syndrome? Klin Wochenschr. 1979;57:181-6.

29. Shirley TL, Lewers JC, Egami K, et al. A human neuronal tissue culture model for Lesch-Nyhan disease. J Neurochem. 2007;101:841-53.

30. Ginzberg MB, Kafri R, Kirschner M. Cell biology. On being the right (cell) size. Science. 2015;348:1245075

31. Dolznig $H$, Grebien $F$, Sauer $T$, et al. Evidence for a size-sensing mechanism in animal cells. Nat Cell Biol. 2004:6:899-905.

32. Glazer HS, Mueller JF, Jarrold T, et al. The effect of vitamin B12 and folic acid on nucleic acid composition of the bone marrow of patients with megaloblastic anemia. J Lab Clin Med. 1954;43:905-13. 